\title{
The microstructure and texture analysis of Ti-6Al-4V alloy through linear friction welding
}

\author{
Zhao Heng $^{1,2, a}$, Liu Qingbin ${ }^{2, b}$, Xie Yaole ${ }^{3, c}$ \\ ${ }^{1}$ Department of Materials, Loughborough University, United Kingdom \\ ${ }^{2}$ National Function Instrument Materials Research Center, \\ Chongqing Materials Research Institute, China \\ ${ }^{3}$ Shen-yang National Laboratory, Institute of Metal Research, China \\ a hengzhao03031989@163.com, ${ }^{b}$ Iqbcq@sohu.com, ${ }^{c}$ xieyaole_9204@126.com
}

Keywords: Ti-6Al-4V alloy, linear friction welding, EBSD (Electron Backscattered Diffraction), BSE (Backscattered Electron) image

\begin{abstract}
The microstructure and texture analysis of Ti-6Al-4V are investigated through SEM (Scanning Electron Microscope). Based on original references, the results show that, the $\beta$ to $\alpha$ phase transformation at the welding center enhances yield strength and ultimate strength through orientations distribution. In detail, the TMAZ (Thermo-Mechanically Affected Zone) around welding line achieves $\alpha$ to $\beta$ phase transformation through SPD (Severe Plastic Deformation) through BSE (Backscattered Electron) images. It is suggested that the phase distribution of $\beta$ phase and $\alpha$ phase is the key to realize orientation distribution and average grain size through EBSD (Electron Backscattered Diffraction). In conclusion, linear friction welding is preferred for Ti-6Al-4V alloy.
\end{abstract}

\section{Introduction}

The linear friction welding attracts the aero-engine industry, such as Rolls-Royce and Boeing, to fabricate titanium alloy for high performance slotted blade assemblies. For the materials selection, the titanium alloy owes low weight, high strength, excellent resistance to corrosion environment among light metals [1]. Furthermore, the low density of titanium behaves not only excellent mechanical performance but also fuel economy with less $\mathrm{CO}_{2}$ emission [2].

Now, the large fun blades which are made of casting Ti-6Al-4V alloy are used instead of polymer materials in Boeing 777 series [3]. At the same time, the Institute of Metal Research, Chinese Academy of Science, actively takes part in Ti-6Al-4V casting alloys manufacturing low pressure turbine blades for Trent series engine for Rolls \& Royce. At the same time, the casting products of Ti-6Al-4V alloy are successfully identified by Rolls \& Royce [4]. On the opposite way, the only manufacture drawbacks are the high price for titanium alloy and limited slip system for deformation behavior [3]. Therefore, shape casting is largely restricted due to limited slip system. Thus, plate welding of titanium alloy is the potential technology into aero-industries.

Linear friction welding shows a great potential in titanium component processing. Linear friction welding is a solid-state component for joining two parts through oscillates one part in linear motion under compressive forces. During welding process, friction heat produces into flashes at the weld edges, sufficient plasticization gradually formed under pressure within several seconds. Thus, clean and high integrity joint is produced which avoids melting and re-solidification processes gives rise to the formation of a narrow heat affected zone (HAZ). In the process of severe formation zone, four distinct phases are distributed in sequence by linear friction welding [5].

In detail, the plastic affected zones are divided into four distinct phases which are evidently distributed from welding zone. It shows as the initial phase, the transition phase, equilibrium phase and deceleration phase, respectively. Firstly, the initial phase: whereby the surface contact area increases due to wear and the thermal soft effect. Secondly, the transition phase: the Heat Affected Zone (HAZ) expands from asperities into the bulk of the material, large wear particles which are 
expelled from the rubbing interface forming initial irregular flash. Thirdly, the equilibrium phase: axial shorten begins as more plasticized matter is expelled forming a uniform flash. Fourthly, the deceleration phase: the short pulse of temperature factor and plastic deformation mutually influence cross-weld mechanical properties and residual stress level through microstructure evolution. In the place of heat affected zone (HAZ) with 4 distinct phases, the $\beta$ phase transforms to $\alpha$ phase at the place of welding positions [6]. However, the phase transformation in these four areas has not been clearly understood.

In order to uncover the nature from $\beta$ to $\alpha$ transformation in the welding line of Ti-6Al-4V alloy, the DRX (Dynamic Recrystallization) and orientation distribution are dominant factors clearly influencing phase transformation.

T.J. Ma et al. studied the fracture behavior of linear friction welded Ti-6Al-4V joints. The microstructure of Ti-6Al-4V linear friction welding region achieves at $61.3 \pm 5.8 \mathrm{~J} / \mathrm{cm}^{2}$ impact toughness. Through fracture morphology investigation, it is believed that the crack develops a short distance along the weld center and TMAZ (Thermo-Mechanically Affected Zone) extending quickly into the parent metal due to weakest impact strength region [7]. Thus, the TMAZ (Thermo-Mechanically Affected Zone) is worthy to be deeply studied through microstructure and mechanical properties.

The TMAZ (Thermo-Mechanically Affected Zone) plays a vital role in the microstructure and fracture behavior of linear friction welding. Which means that, the DRX (Dynamic Recrystallization) generally soft the TMAZ (thermo-mechanically affected zone). Hence, the equiaxed crystal grains behave a lower hardness than base material with higher joint efficiency, less distortion and lower residual stress [8].

L. Zhou et al. found that, the $\beta$ transform to $\alpha$ from welding region through DRX (Dynamic Recrystallization). The base material which constructs with $\alpha$ phase and $\beta$ phase transforms to dominant $\alpha$ lamellar fine structure with $\beta$ phase under determined temperature. In summary, the primary $\alpha$ lamellar fine structure shows a rather lower hardness than a combination of $\alpha$ phase and $\beta$ phase for base material with a higher hardness. In other words, the DRX (Dynamic Recrystallization) functions soften microstructure which leads residual stress and hardness decrease [9].

On the opposite way, the SPD (Severe Plastic Deformation) leads to a high residual stress and high strength at the welding center. Similarly, J. Romero et al. revealed that the linear friction welding creates such a steep thermal gradient that high residual stress is occurred. The higher content of $\beta$ phase leads to a higher hardness at the same level of temperature which is identified in this experiment [9]. For example, the Ti-6Al-4V series alloy provided by Rolls \& Royce realize the yield strength of $960 \mathrm{MPa}$ and $1050 \mathrm{MPa}$, the ultimate strength of $1050 \mathrm{MPa}$ and $930 \mathrm{MPa}$ for D-Ti-64 and B-Ti-64, respectively. At the same time, the hardness the hardness around weld center reaches approximately 420-430 $\mathrm{HV}$ which is higher than base material of approximately $344 \mathrm{HV}$ for Di-Ti-64 alloy and approximately $328 \mathrm{HV}$ for B-Ti-64 alloy [10].

In summary, the $\beta$ to $\alpha$ phase transformation through DRX (Dynamic Recrystallization) achieves lower hardness around welding center. On the other hand, a higher content of $\beta$ phase achieve higher hardness with rather high residual stress and yield strength. However, the factors, such as texture influences, have not been taken into considerations. In order to uncover the nature of $\beta$ to $\alpha$ phase transformation, the Electron Backscattered Diffraction (EBSD) is used for analysis and measurement.

\section{Experimental Procedure}

Material Processing. The Ti-6Al-4V alloy components are provided by Rolls\&Rocye, Derby. The size of Ti-6Al-4V block is $30 \mathrm{~mm} \times 40 \mathrm{~mm} \times 10 \mathrm{~mm}$. Two different heat treatment Ti-6Al-4V alloys are manufactured as blade and disk, respectively. Before linear friction welding, argon shielding is used to avoid any contaminations such as the presence of hydrogen, oxygen, nitrogen or carbon contamination. The schematic diagram of linear friction welding is shown in the figure 1 [11]. After linear friction welding happens, the microstructure of Ti-6Al-4V component is analyzed 
through SEM (Scanning Electron Microscopy).
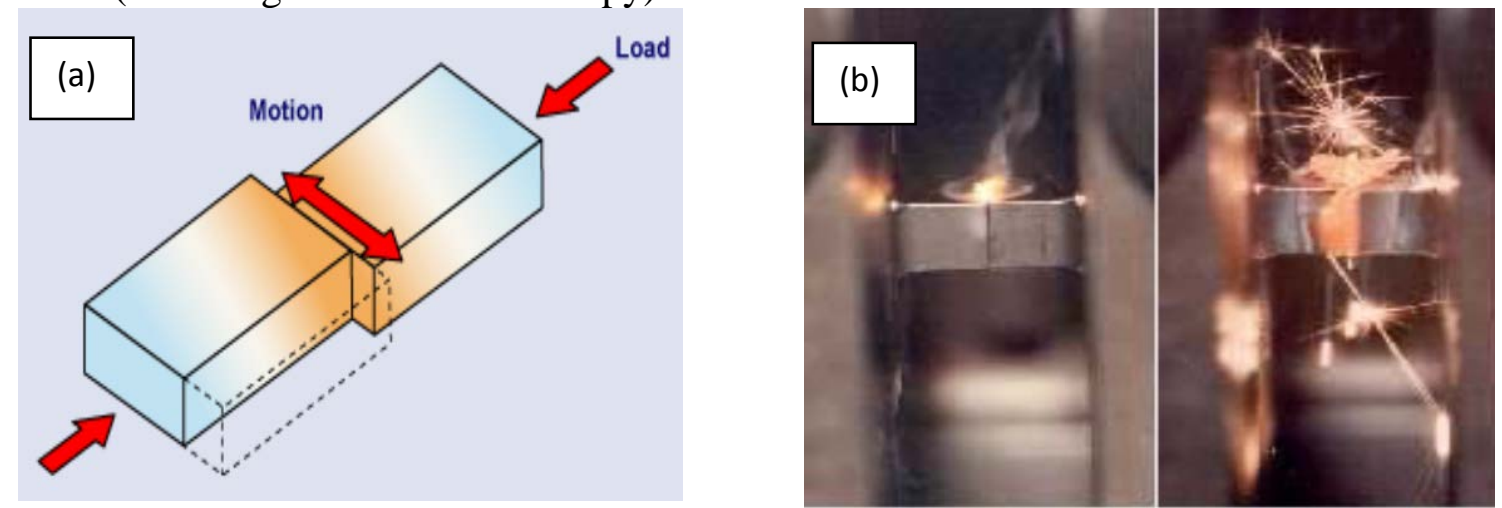

Figure 1. the schematic of linear friction welding (a) and linear friction of titanium (b) [11]

Material Characterization. The Backscattered Electron images and Electron Backscattered Diffraction images are conducted through SEM (Scanning Electron Microscope). In detail, the enlarged section polished using semi-automatic polishing machine (TegraPol-25, Struers Ltd., Solihull, United Kingdom) with successively finer diamond polish disk. Further polishing explains that, the ultra-fine grain size is achieved by $3 \mathrm{M}^{\#}$ Diamond Grinding Paper and Struers Oxide Polish Suspension Compound. And then, the analyzed SEM (Leo 1530 VP, Field Emission Gun Scanning Electron Microscope, LEO Elektroneskopie GmbH, Oberkochen, Germany) is used to collect effective information on plastic affected zone or thermo-mechanically zone from Ti-6Al-4V samples.

\section{Results and Discussions}

In order to achieve high hardness and excellent yield strength, the microstructure of $\beta$ phase dominate in Ti-6Al-4V alloy in the samples which are provided by Rolls \& Royce for aircraft manufacture. The excellent yield strength and hardness both are achieved through $\beta$ phase distribution in the figure 2.
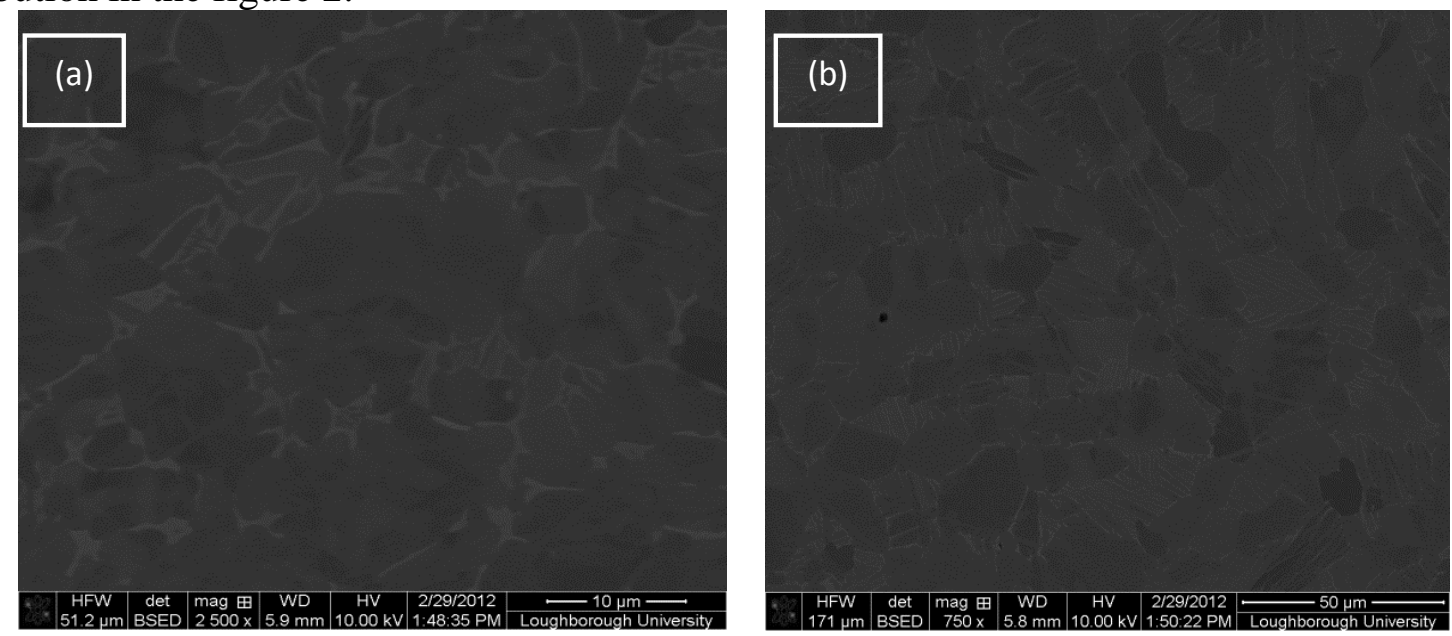


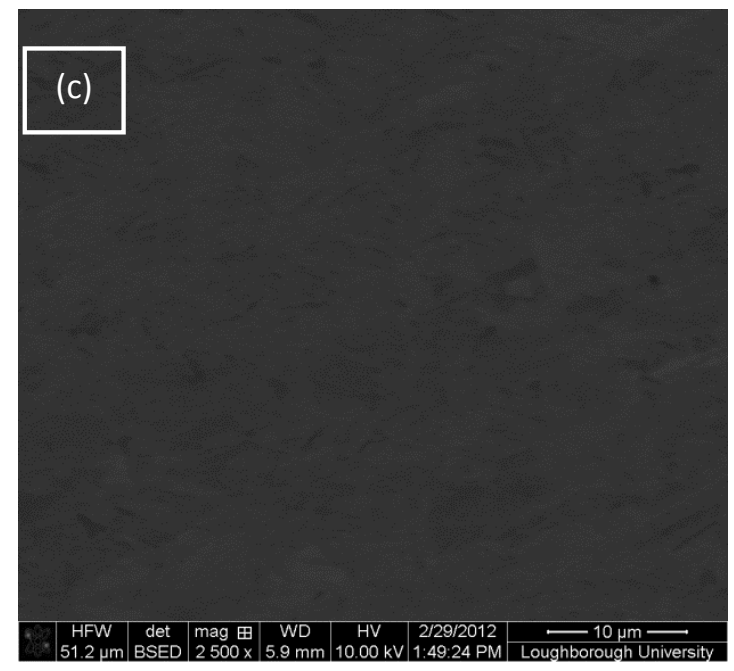

Figure 2. The microstructure of blade (a), disk (b), weld line (c), through BSE (Backscattered Electron) images, respectively. Supplement: The BSE (Backscattered Electron) images are used to differentiate phase identification.

Figure 3 shows that the orientation maps and corresponding pole figures of the blade, disc and weld line, independently. As for the blade in Figure 3 (a), the orientation of the dominant $\alpha$ grain is very random. The $\beta$ phase is located along grain boundaries of $\alpha$ phase. In the figure 3 (b), the texture distribution is random without regularity.
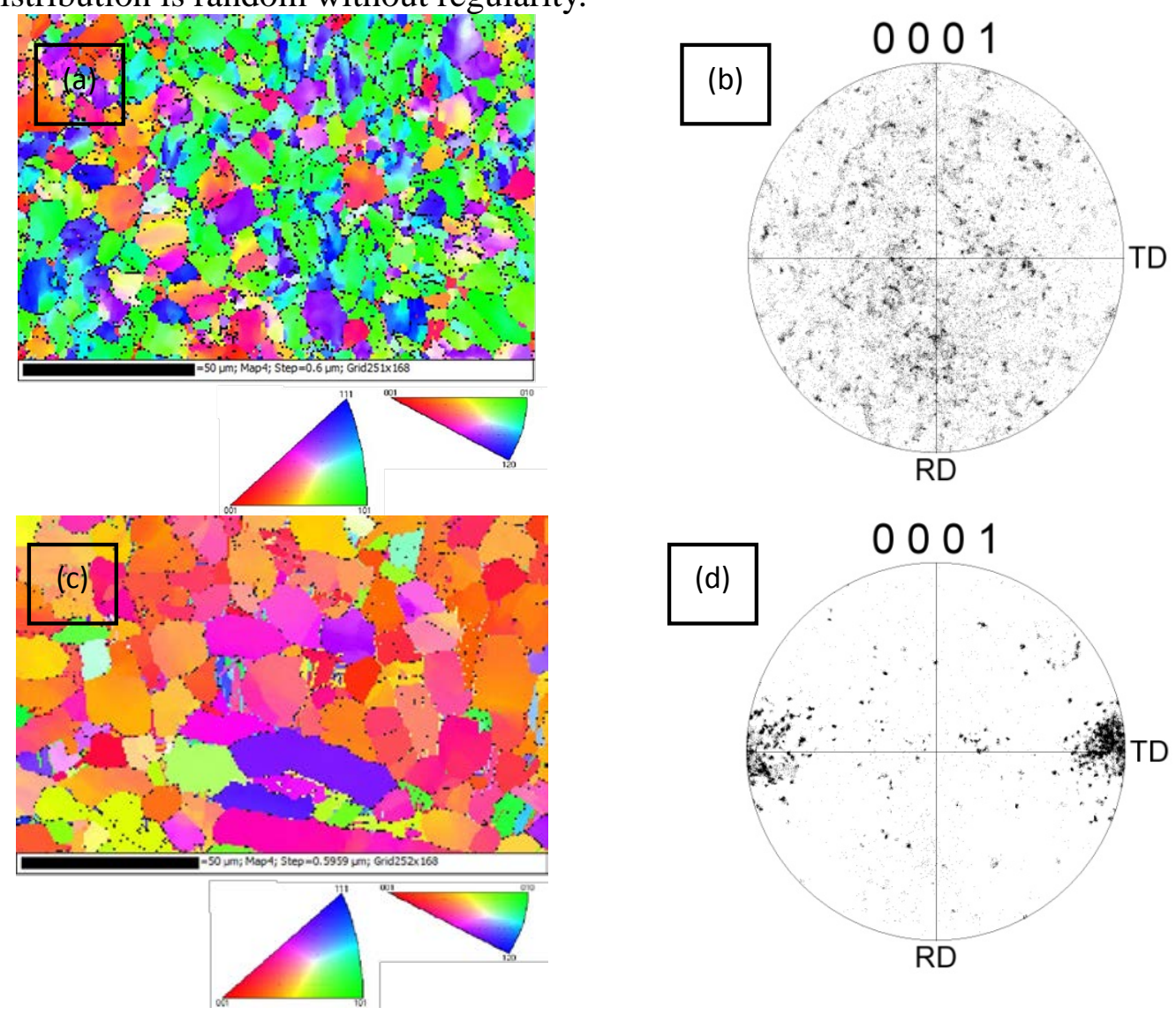

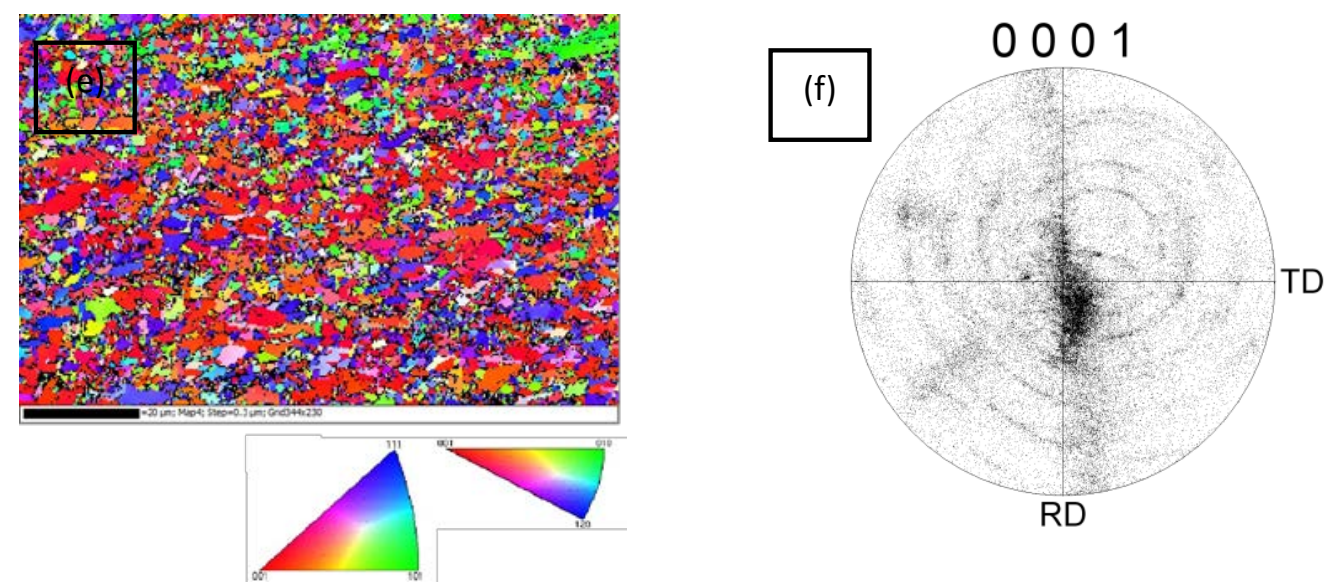

Figure 3. The EBSD (Electron Backscattered Diffraction) of linear stir friction: the left side is EBSD (Electron backscattered Diffraction) images of blade (a), disk (c) and weld line (e), respectively. The right sides are pole figures, which represent to blade (b), disk (d) and weld line (f), respectively.

In terms of the disc in Figure 3 (c) (d), it is clear that most of $\alpha$ grains shows a preferred orientation from [ $\left[\begin{array}{llll}0 & 0 & 0 & 1\end{array}\right]$ direction at the transverse direction. The sizes of $\alpha$ grain are from $10 \mathrm{~nm}$ to $32 \mathrm{~nm}$. And, $\beta$ phases are approximately $1 \mathrm{~nm}$ located along grain boundaries of $\alpha$ phase.

In Figure 3 (e) (f), most of the grains in the weld line has a preferred orientation at $\left[\begin{array}{llll}0 & 0 & 0 & 1\end{array}\right]$ which is normal direction. The grains are much smaller than that of the blade and disc. Specifically, the TMAZ (Thermo-Mechanically Affected Zone) around fine structure in the center of weld line presents $\alpha$ to $\beta$ phase transformation in figure 2 (b). As the compressive force along linear motion achieves SPD (Severe Plastic Deformation), high yield strength and ultimate strength in consistence orientation could be realized by rapid increasing $\beta$ phases without DRX (Dynamic Recrystallization) [10].

Hence, from the figure 3 (a) (c) (e), the fine grain distribution is achieved in weld line figure 3 (e) with compare to figure 3 (a) (c). At the same time, the pole figure 3 (f) achieves consistence orientation in contrast to the pole figure 3 (b) (d). Comprehensively, the texture distribution still disperses randomly at several points which enhance mechanical properties of Ti-6Al-4V alloy on vary directions [10]. Furthermore, it could prevent weakest crack originality as well as enhance impact toughness. As a whole, a preferable orientation offers consistence deformation behavior which could prevent crack origins [6].

\section{Conclusions}

1. It is fundamentally explained by BSE (Backscattered Electron) images that, the $\beta$ to $\alpha$ phase transformation through DRX (Dynamic Recrystallization) achieves lower hardness around welding center. On the other hand, a higher content of $\beta$ phase achieve higher hardness with high residual stress and yield strength.

2. In case of the mechanical properties of linear friction welding, the TMAZ (Thermo-Mechanically Affected Zone) around welding line achieves $\alpha$ to $\beta$ phase transformation through SPD (Severe Plastic Deformation) in order to acquire excellent mechanical properties.

3. The dominant factors of consistence orientation distribution owe deformation behavior and disperse texture distribution which prevents crack origins along TMAZ (Thermo-Mechanically Affected Zone).

\section{Acknowledgements}

The authors are grateful to Department of Materials, Loughborough University. Specifically, they 
are thankful to Yuan Hefeng and Dr. Simon Hogg who provide original statistics and the instruction for titanium processing, respectively. At the same time, this article is indirectly supported by National Nature Science Foundation of China (51331008). To the last, the technical comment is offered from Shen-Yang National Laboratary, Institute of Metal Research, Chinese Academy of Sciences.

\section{References}

[1] S. Hogg and T.YauYau, in: Metals Processing and Properties, edited by Loughborough University, United Kingdom (2013), p. 83-84 Unpublished.

[2] R. Turner, J.C. Gebelin, R.M. Ward, R.C. Reed: Acta Mater. , Vol. 59 (2011), p. 3792-3803

[3] G. Lutjering and J.C. Williams, in: Titanium (2nd edition)-chapter 10: Special Properties and Applications of Titanium, edited by Springer Science+Business Media, NewYork, United States of American (2007) , p. 396-399;1,19-21.

[4] Information on: Institute of Metal research, Shenyang, Chinese Science Academy An engineer named Simon Weeks from Rolls-Royce Company is invited to Institute of Metal Research on Aircraft-manufacture Corporation. $\quad$ Retrieved 30th June, 2013 from http://www.imr.cas.cn/xwzx/zhxw/201305/t20130502_3830354.html

[5] S. Hoggs, in: Metals Processing and Properties for Laboratory Workshop-Microstructure of linear friction welded Ti-6Al-4V alloy, edited by Loughborough University, United Kingdom (2013) Unpublished Sheet.

[6] M. Karadge, M. Preuss, C. Lovell, P.J. Withers and S. Bray: Mater. Sci. Eng., A Vol. 459 (2007), p. $182-191$

[7] T.J. Ma, W.Y. Li and S.Y. Yang: Mater. Des. Vol. 30 (2009), p. 2131

[8] L.H. Wu, D. Wang, B.L. Xiao and Z.Y. Ma: Scripta Mater. Vol. 78-79 (2014), p. 17-20

[9] L. Zhou, H.J. Liu, P. Liu and Q.W. Liu: Scripta Mater. Vol. 61 (2009), p. 596-599

[10] J. Romero, M.M. Attallah, M. Preuss, M. Karadge and S.E. Bray: Acta Mater. Vol. 57 (2009), p. 5583-5586

[11] Information on: Philip Threadgill from TWI http://www.twi.co.uk/. 